\title{
Enhancement of thermoelectric effects in a single mo- lecular magnet due to resonance tunnelling
}

\author{
Jian-Hua Wang ${ }^{1}$, Wei-Ping Xu' ${ }^{1}$, Yu-Ying Zhang ${ }^{2}$, Yi-Hang Nie ${ }^{1, \text { * }}$ \\ ${ }^{1}$ Institute of Theoretical Physics, Shanxi University, Taiyuan, China \\ ${ }^{2}$ Department of Physics, Shanxi University, Taiyuan, China
}

Email address:

nieyh@sxu.edu.cn(Yi-Hang Nie)

\section{To site this article:}

Jian-Hua Wang, Wei-Ping Xu, Yu-Ying Zhang, Yi-Hang Nie. Enhancement of Thermoelectric Effects in a Single Molecular Magnet due to Resonance Tunnelling. American Journal of Modern Physics. Vol. 3, No. 2, 2014, pp. 37-44. doi: 10.11648/j.ajmp.20140302.12

\begin{abstract}
Thermoelectric properties of the single molecular magnet coupled to two metal electrodes are investigated theoretically. For the infinite Coulomb interaction the singly-occupied states determine the effective transport channels. At the low temperature the thermopower and the figure of merit present the large values near bonding state level, and for the given temperature the thermopower and the figure of merit are significantly enhanced with coupling intensity decreasing. When Coulomb interaction is finite, the spectra of thermoelectric quantities split into two sets. The peaks of the thermopower and figure of merit corresponding to doubly-occupied transport channels are much larger than unity for the small coupling intensity. These results are useful for understanding and designing highly efficient thermoelectric devices based on molecular magnet.
\end{abstract}

Keywords: Molecular Magnet, Thermoelectric Effect, Coulomb Blockade

\section{Introduction}

Thermoelectric materials can directly convert the thermal energy into the electrical energy. Although the thermoelectric phenomenon has been known for long time, its efficiency in bulk materials was relatively low and thus the energy conversion technology based on the thermoelectric materials has not been widely used. The thermoelectric effects of material originate from the asymmetry of the electrons and holes near the Fermi level. The efficiency of a thermoelectric material is depicted by the dimensionless thermoelectric figure of merit, $\mathrm{ZT}=\mathrm{GS}_{\mathrm{e}}^{2} \mathrm{~T} / \kappa$, where $\mathrm{S}_{\mathrm{e}}$ is the Seebeck coefficient (thermopower), $G$ is the electric conductance, $\kappa$ is the thermal conductance and $T$ is the temperature of the device. In conventional solid materials, $\mathrm{G}$ and $\kappa$ obey the Wiedemann-Franz law $(\kappa / \mathrm{GT}=$ $\left.\left(\mathrm{k}_{\mathrm{B}} \pi\right)^{2} / 3 \mathrm{e}^{2}\right)$ [1], which means that an increase in the electric conductance also leads to an increase in the thermal conductance for a given temperature. At the same time, according to the Mott relation $\left(\mathrm{S}_{\mathrm{e}}=-\mathrm{eL}_{0} \mathrm{G}^{-1} \partial \mathrm{G} / \partial \mathrm{E}_{\mathrm{F}}\right)$ [2], an increase in the electric conductance is apt to lead to a decrease in Seebeck coefficient. Thus the maximum ZT of the conventional bulk material has been holding at about 1 , which restricts the wide application of thermoelectric material in industry.
With the development of nanotechnology, the study of thermoelectric effect enters the era of the nanoscale. Due to the quantum effects such as level quantization and Coulomb blockade emerged in the nano systems, the Mott relation and the Wiedemann-Franz law may not hold in the nanostructures [3, 4], and the thermoelectric properties of the nanostructures attract much attention experimentally and theoretically [5-15]. For example, Coulomb blockade oscillations of the thermopower and the thermal conductance were obtained for Coulomb islands with the gate voltage [9]. In multilevel QD systems the bipolar effect has been observed in the thermal conductance spectrum at high temperature [4]. Moreover, in low dimensional materials the lattice thermal conductance can be significantly reduced. As a result, the thermoelectric figure of merit in nanostructure such as quantum dot and nanotube can arrive at the value of ZT > $1[4,16]$. Specially, in single-molecule devices the high figure of merit (ZT $\gg 1$ ) can be obtained by tuning Fano resonances near the Fermi energy [17]. The similar conclusions have been confirmed in double quantum dot molecular junction $[18,19]$. Since the thermoelectric effect in molecular junction can reveal transport mechanisms through the molecular junction [20] and the experiments have demonstrated the capability of measuring the Seebeck coefficient in atomic and molecular junctions [21, 
22], the thermoelectric effect of the single molecule devices attracts much interest [5, 17, 23-27].

Single molecular magnet (SMM), characterized by a large spin and magnetic anisotropy, has attracted much attention due to showing many unique transport properties. These transport properties result from the correlation between the charge states and spin states of SMM, which can lead to the better thermoelectric figure of merit than the conventional bulk material. Recently, it is found the intrinsic magnetic anisotropy of SMM can lead to gate voltage dependent oscillations of charge thermopower and a large violation of the Wiedeman-Franz law, but a pure spin thermopower can be obtained by tuning only the gate voltage [25]. Moreover, in a single molecular magnet tunnel junction the highly spin-polarized current can be generated by thermoelectric effects and its flowing direction and spin polarization can be changed by adjusting the gate voltage applied to the SMM [26]. The SMM nowadays has become a good candidate for molectronics and spintronics devices. The theoretical study of the thermoelectricity in the molecule magnet is helpful for understanding the relation between quantum mechanics and thermal phenomena; moreover, it may predict the application prospect of the molecule magnet.

In this paper we study thermoelectric effects in an isotropic SMM connected to two metal electrodes in the linear response regime. By using the non-equilibrium Green function technique based on Hubbard operator, we present the thermopower and the figure of merit as functions of the temperature and the coupling intensity between the SMM and the electrodes and give the optimized parameter region of the figure of merit, where the figure of merit can be much larger than that in conventional bulk materials.

\section{Model and Formalism}

The SMM coupled to two metal electrodes can be described by the Hamiltonian proposed by Timm and Elste [28]. The system considered here consists of an isotropic molecular magnet coupled to two metal electrodes and also can be described by a similar Hamiltonian,

$$
\mathrm{H}=\mathrm{H}_{\text {leads }}+\mathrm{H}_{\mathrm{T}}+\mathrm{H}_{\text {SMM }},
$$

where the first term, $\mathrm{H}_{\text {leads }}=\sum_{\mathrm{k} \alpha \sigma} \epsilon_{\mathrm{k} \alpha} \mathrm{c}_{\mathrm{k} \alpha \sigma}^{+} \mathrm{c}_{\mathrm{k} \alpha \sigma}$, describes the noninteracting electrons in the metallic leads, where $\mathrm{c}_{\mathrm{k} \alpha \sigma}^{+}$ creates an electron with spin $\sigma$, momentum $\mathrm{k}$ and energy $\epsilon_{\mathrm{k} \alpha}$ in the lead $\alpha=\mathrm{L}, \mathrm{R}$. The second term, $\mathrm{H}_{\mathrm{T}}=$ $\sum_{\mathrm{k} \alpha \sigma}\left(\mathrm{t}_{\mathrm{k} \alpha} \mathrm{c}_{\mathrm{k} \alpha \sigma}^{+} \mathrm{c}_{\sigma}+\right.$ H. c. $)$, represents the coupling between the SMM and the leads with $t_{\mathrm{k} \alpha}$ the hopping-matrix element. In the paper, we only consider the isotropic SMM which is described by the third term in equation (1) and its unperturbed Hamiltonian can be expressed as

$$
\mathrm{H}_{\mathrm{SMM}}=\sum_{\sigma} \epsilon_{0} \hat{\mathrm{n}}_{\sigma}+\mathrm{U} \hat{\mathrm{n}}_{\uparrow} \hat{\mathrm{n}}_{\downarrow}-\mathrm{Js} \cdot \mathrm{S},
$$

here $\epsilon_{0}=\epsilon-\mathrm{eV}_{\mathrm{g}}$ is the lowest unoccupied molecular orbit (LUMO) energy which can be tuned by the gate vol- tage $V_{g}, \hat{n}_{\sigma}=c_{\sigma}^{+} c_{\sigma}(\sigma=\uparrow, \downarrow)$ is the number operator, where $c_{\sigma}^{+}$creates an electron with energy $\epsilon$ in the molecular orbit. $\mathrm{s}=\sum_{\sigma \sigma^{\prime}} \mathrm{c}_{\sigma}^{+}\left(\tau_{\sigma \sigma^{\prime}} / 2\right) \mathrm{c}_{\sigma^{\prime}}$ is the corresponding spin operator with $\tau_{\sigma \sigma^{\prime}}$ the Pauli matrix elements. $U$ is the Coulomb charging energy and $\mathrm{J}$ is the exchange interaction between an electron in the orbit and the local spin $S \geq 1$. $S^{z}$ is the $\mathrm{z}$ component of the giant spin operator $\mathrm{S}$. The exchange interaction term $\mathrm{Js} \cdot \mathrm{S}$ can be rewritten as $\mathrm{Js}^{\mathrm{Z}} \mathrm{S}^{\mathrm{Z}}+\mathrm{Js}^{+} \mathrm{S}^{-} / 2+\mathrm{Js}^{-} \mathrm{S}^{+} / 2$ with $\mathrm{S}^{ \pm}=\mathrm{S}^{\mathrm{x}} \pm \mathrm{iS}^{\mathrm{y}}$ (similarly for $s^{ \pm}$) the spin-flip operators.

The eigenstates of the unperturbed SMM can be expressed as $|n, m\rangle$ with $n=0,1,2$ electrons and the $\mathrm{z}$ component of the total spin $\mathrm{m}$ [28]. In terms of the electron spin states $|\sigma\rangle(\sigma=0, \uparrow, \downarrow, \uparrow \downarrow)$ in the molecular orbital and the local spin states $|\mathrm{m}\rangle \quad(\mathrm{m} \in[-\mathrm{S}, \mathrm{S}])$, one can reads $|0, \mathrm{~m}\rangle=|0\rangle \otimes|\mathrm{m}\rangle$ with $\epsilon_{0, \mathrm{~m}}=0$ for $\mathrm{n}=0$ and $|2, \mathrm{~m}\rangle$ $=|\uparrow \downarrow\rangle \otimes|\mathrm{m}\rangle$ with $\epsilon_{2, \mathrm{~m}}=2\left(\epsilon-\mathrm{eV}_{\mathrm{g}}\right)+\mathrm{U}$ for $\mathrm{n}=2$. For $\mathrm{n}=1$, two orthogonal singly-occupied states read

$$
|1, \mathrm{~m}\rangle^{ \pm}=\mathrm{A}_{\mathrm{m}}^{ \pm}|\downarrow\rangle \otimes|\mathrm{m}+1 / 2\rangle+\mathrm{B}_{\mathrm{m}}^{ \pm}|\uparrow\rangle \otimes|\mathrm{m}-1 / 2\rangle
$$

with energies $\epsilon_{1, \mathrm{~m}}^{ \pm}=\epsilon-\mathrm{eV}_{\mathrm{g}}+1 / 4 \pm \Delta \mathrm{E}(\mathrm{m} \in[-\mathrm{S}+$ $1 / 2, \mathrm{~S}-1 / 2])$ and $\Delta \mathrm{E}=\mathrm{J}(2 \mathrm{~S}+1) / 4$. It is obvious that the molecular states $|1, \mathrm{~m}\rangle^{ \pm}$are degenerate due to the absence of anisotropy and external magnetic field. The coefficients $A_{\mathrm{m}}^{ \pm}=\mp \frac{\sqrt{2 \Delta \mathrm{E} \pm J \mathrm{~m}}}{2 \sqrt{\Delta \mathrm{E}}}$ and $B_{\mathrm{m}}^{ \pm}=\frac{\mathrm{J} \sqrt{\mathrm{S(S+1)- \textrm {m } ^ { 2 } + 1 / 4}}}{2 \sqrt{\Delta \mathrm{E}} \sqrt{2 \Delta \mathrm{E} \pm \mathrm{J}}}$. For the fully polarized states $|1,-S-1 / 2\rangle$ and $|1, S+1 / 2\rangle$, the energies is $\epsilon_{1, \pm(S+1 / 2)}=\epsilon-\mathrm{eV}_{\mathrm{g}}-\mathrm{JS} / 2$ for $\mathrm{m}= \pm(\mathrm{S}+1)$.

According to the nonequilibrium Green function approach, the electric and heat currents flowing from the left to the right lead are formulized as [26, 29-31]

$$
\begin{gathered}
I=-\frac{\mathrm{e}}{\mathrm{h}} \sum_{\sigma} \int\left[\mathrm{f}_{\mathrm{L}}(\omega)-\mathrm{f}_{\mathrm{R}}(\omega)\right] \Gamma \operatorname{ImG}_{\sigma}^{\mathrm{r}}(\omega), \\
\mathrm{I}_{\mathrm{Q}}=-\frac{1}{\mathrm{~h}} \sum_{\sigma} \int\left(\omega-\mu_{\mathrm{L}}\right)\left[\mathrm{f}_{\mathrm{L}}(\omega)-\mathrm{f}_{\mathrm{R}}(\omega)\right] \Gamma \operatorname{ImG} \mathrm{G}_{\sigma}^{\mathrm{r}}(\omega),
\end{gathered}
$$

where $\mathrm{f}_{\alpha}(\omega)=\left[\mathrm{e}^{\left(\omega-\mu_{\alpha}\right) / \mathrm{k}_{\mathrm{B}} \mathrm{T}_{\alpha}}+1\right]^{-1}$ is the Fermi distribution function with temperature $\mathrm{T}_{\alpha}$ and chemical potential $\mu_{\alpha}$ in the lead $\alpha . G_{\sigma}^{r}(\omega)=\left\langle\left\langle c_{\sigma} \mid c_{\sigma}^{+}\right\rangle\right\rangle^{r}$ is the Fourier transform of the retarded Green function $\mathrm{G}_{\sigma}^{\mathrm{r}}(\omega)=$ $-i \theta(t)\left\langle\left\{c_{\sigma}(t), c_{\sigma}^{+}(0)\right\}\right\rangle$. In the following calculation, we consider symmetric barriers, i.e., $\Gamma=\Gamma_{L}=\Gamma_{R}=2 \pi|t|^{2} \rho$ and $\Gamma_{\uparrow}=\Gamma_{\downarrow}$, where $\rho=1 / 2 \mathrm{D}$ is the density of states of leads for the square band approximation and $\mathrm{D}$ is the half bandwidth.

In order to calculate expediently the retarded Green function, the total Hamiltonian (1) is rewritten by means of Hubbard operators [31] as

$$
\begin{gathered}
\mathrm{H}=\sum_{\mathrm{k} \alpha \sigma} \epsilon_{\mathrm{k} \alpha} \mathrm{c}_{\mathrm{k} \alpha \sigma}^{+} \mathrm{c}_{\mathrm{k} \alpha \sigma}+\sum_{\mathrm{k} \alpha \sigma}\left[\mathrm{t}_{\mathrm{k} \alpha} \mathrm{c}_{\mathrm{k} \alpha \sigma}^{+}\left(\mathrm{X}^{0 \sigma}+\delta_{\sigma} \mathrm{X}^{\bar{\sigma} 2}\right)\right. \\
+ \text { H. c. }]+\sum_{\sigma=\uparrow, \downarrow} \epsilon_{0} \mathrm{X}^{\sigma \sigma}+\left(2 \epsilon_{0}+\mathrm{U}\right) \mathrm{X}^{22}
\end{gathered}
$$




$$
\begin{gathered}
-\frac{J}{2} \sum_{m=-S}^{S} m X^{\uparrow \uparrow} Y^{m m}+\frac{J}{2} \sum_{m=-S}^{S} m X^{\downarrow \downarrow} Y^{m m} \\
-\frac{J}{2} \sum_{m=-S}^{S} C_{m}^{-} X^{\uparrow \downarrow} Y^{m-1, m}-\frac{J}{2} \sum_{m=-S}^{S} C_{m}^{+} X^{\downarrow \uparrow} Y^{m+1, m}
\end{gathered}
$$

where $\epsilon_{0}=\epsilon-\mathrm{eV}_{\mathrm{g}}, \delta_{\sigma}=+1(-1)$ for $\sigma=\uparrow(\downarrow)$ ( $\bar{\sigma}$ is opposite to $\sigma$ ). In the equation (6) the electron operators in molecular orbital and the local spin operator in magnet have be expressed as the Hubbard operators, i.e., $c_{\sigma}=X^{0 \sigma}$, $+\delta_{\sigma} \mathrm{X}^{\bar{\sigma} 2}, \quad \mathrm{c}_{\sigma}^{+}=\mathrm{X}^{\sigma 0}+\delta_{\sigma} \mathrm{X}^{2 \bar{\sigma}}, \quad \mathrm{s}^{+}=\mathrm{X}^{\uparrow \downarrow}, \quad \mathrm{s}^{-}=\mathrm{X}^{\downarrow \uparrow}$, $\mathrm{s}^{\mathrm{z}}=\left(\mathrm{X}^{\uparrow \uparrow}-\mathrm{X}^{\downarrow \downarrow}\right) / 2$, where the Hubbard operators $\mathrm{X}^{\sigma \sigma^{\prime}}$ are defined in terms of the electron spin states $|\sigma\rangle$ with $\mathrm{X}^{\sigma \sigma^{\prime}}=|\sigma\rangle\left\langle\sigma^{\prime}\right|$, satisfying the closure relation $\sum_{\sigma} X^{\sigma \sigma}=1$. Similarly, the local large spin operators may be expressed as

$$
\begin{aligned}
& \mathrm{S}^{\mathrm{z}}=\sum_{\mathrm{m}=-\mathrm{S}}^{\mathrm{S}} \mathrm{m} \mathrm{Y}^{\mathrm{m}, \mathrm{m}}, \\
& \mathrm{S}^{+}=\sum_{\mathrm{m}=-\mathrm{S}}^{\mathrm{S}} \mathrm{C}_{\mathrm{m}}^{+} \mathrm{Y}^{\mathrm{m}+1, \mathrm{~m}}, \\
& \mathrm{~S}^{-}=\sum_{\mathrm{m}=-\mathrm{S}}^{\mathrm{S}} \mathrm{C}_{\mathrm{m}}^{-} \mathrm{Y}^{\mathrm{m}-1, \mathrm{~m}},
\end{aligned}
$$

where $\mathrm{Y}^{\mathrm{mm}} \mathrm{m}^{\prime} \equiv|\mathrm{Sm}\rangle\left\langle\mathrm{Sm}^{\prime}\right|, \mathrm{C}_{\mathrm{m}}^{ \pm} \equiv \sqrt{(\mathrm{S} \pm \mathrm{m}+1)(\mathrm{S} \mp \mathrm{m})}$ and $\sum_{\mathrm{m}} \mathrm{Y}^{\mathrm{mm}}=1$. The retarded Green function based on Hubbard operator representation can be rewritten as

$$
\begin{gathered}
\mathrm{G}_{\sigma}^{\mathrm{r}}(\omega)=\left\langle\left\langle\mathrm{X}^{0 \sigma} \mid \mathrm{c}_{\sigma}^{+}\right\rangle\right\rangle^{\mathrm{r}}+\delta_{\sigma}\left\langle\left\langle\mathrm{X}^{\bar{\sigma} 2} \mid \mathrm{c}_{\sigma}^{+}\right\rangle\right\rangle^{\mathrm{r}} \\
=\sum_{\mathrm{m}}\left\langle\left\langle\mathrm{X}^{0 \sigma} \mathrm{Y}^{\mathrm{mm}} \mid \mathrm{c}_{\sigma}^{+}\right\rangle\right\rangle^{\mathrm{r}}+\delta_{\sigma} \sum_{\mathrm{m}}\left\langle\left\langle\mathrm{X}^{\bar{\sigma} 2} \mathrm{Y}^{\mathrm{mm}} \mid \mathrm{c}_{\sigma}^{+}\right\rangle\right\rangle^{\mathrm{r}} .
\end{gathered}
$$

In the sequential tunneling regimes, by employing the equation of motion [32] and adopting second-order approximation [33], the retarded Green function appearing on the left-hand side of equation (10) is expressed as

$$
\begin{aligned}
& \left\langle\left\langle\mathrm{X}^{0 \sigma} \mathrm{Y}^{\mathrm{mm}} \mid \mathrm{c}_{\sigma}^{+}\right\rangle\right\rangle^{\mathrm{r}} \\
& =\frac{\alpha_{\mathrm{m} \pm 1 / 2}^{ \pm}\left(\mathrm{P}_{0 \mathrm{~m}}+\mathrm{P}_{\sigma \mathrm{m}}\right)-\beta_{\mathrm{m} \pm 1 / 2}\left\langle\mathrm{X}^{\sigma \bar{\sigma}} \mathrm{Y}^{\mathrm{m}, \mathrm{m} \pm 1}\right\rangle}{\omega-\Delta_{|1, \mathrm{~m} \pm 1 / 2\rangle^{+} \rightarrow|0, \mathrm{~m}\rangle}-\Sigma_{0}} \\
& +\frac{\alpha_{\mathrm{m} \pm 1 / 2}^{\mp}\left(\mathrm{P}_{0 \mathrm{~m}}+\mathrm{P}_{\sigma \mathrm{m}}\right)+\beta_{\mathrm{m} \pm 1 / 2}\left\langle\mathrm{X}^{\sigma \bar{\sigma}} \mathrm{Y}^{\mathrm{m}, \mathrm{m} \pm 1}\right\rangle}{\omega-\Delta_{|1, \mathrm{~m} \pm 1 / 2\rangle^{-} \rightarrow|0, \mathrm{~m}\rangle^{-\Sigma_{0}}}} \\
& \left\langle\left\langle\mathrm{X}^{\bar{\sigma} 2} \mathrm{Y}^{\mathrm{mm}} \mid \mathrm{c}_{\sigma}^{+}\right\rangle\right\rangle^{\mathrm{r}} \\
& =\frac{\alpha_{\mathrm{m} \mp 1 / 2}^{\mp} \delta_{\sigma}\left(\mathrm{P}_{\bar{\sigma} \mathrm{m}}+\mathrm{P}_{2 \mathrm{~m}}\right)-\beta_{\mathrm{m} \mp 1 / 2} \delta_{\sigma}\left\langle\mathrm{X}^{\sigma \bar{\sigma}} \mathrm{Y}^{\mathrm{m} \mp 1, \mathrm{~m}}\right\rangle}{\omega-\Delta_{|2, \mathrm{~m}\rangle \rightarrow|1, \mathrm{~m} \mp 1 / 2\rangle^{+}}-\Sigma_{0}}
\end{aligned}
$$

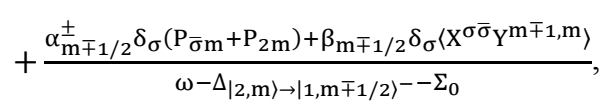

where we define the self-energy $\Sigma_{0}=\frac{\sum_{\mathrm{k} \alpha}\left|\mathrm{t}_{\mathrm{k} \alpha}\right|^{2}}{\omega-\epsilon_{\mathrm{k} \alpha}+\mathrm{i} 0^{+}}=-\mathrm{i} \Gamma$, the factor $\alpha_{\mathrm{m}}^{ \pm}=\frac{\mp \mathrm{Jm} / 2+\Delta \mathrm{E}}{2 \Delta \mathrm{E}}$ with $\alpha_{\mathrm{m}}^{+}+\alpha_{\mathrm{m}}^{-}=1$ and $\beta_{\mathrm{m}}=$ $\frac{\mathrm{J}}{4 \Delta \mathrm{E}}\left[(\mathrm{S}+1 / 2)^{2}-\mathrm{m}^{2}\right]^{1 / 2}$. The averages $\mathrm{P}_{\sigma \mathrm{m}}$ are defined as $\mathrm{P}_{\sigma \mathrm{m}}=\left\langle\mathrm{X}^{\sigma \sigma} \mathrm{Y}^{\mathrm{mm}}\right\rangle(\sigma=0, \uparrow, \downarrow, 2)$ and the energy differences are written as $\Delta_{|1, \mathrm{~m} \pm 1 / 2\rangle^{+} \rightarrow|0, \mathrm{~m}\rangle}=\epsilon_{1, \mathrm{~m} \pm 1 / 2}^{+}-$ $\epsilon_{0, \mathrm{~m}}, \Delta_{|1, \mathrm{~m} \pm 1 / 2\rangle^{-} \rightarrow|0, \mathrm{~m}\rangle}=\epsilon_{1, \mathrm{~m} \pm 1 / 2}^{-}-\epsilon_{0, \mathrm{~m}}, \Delta_{|2, \mathrm{~m}\rangle \rightarrow|1, \mathrm{~m} \mp 1 / 2\rangle^{+}}=$ $\epsilon_{2, \mathrm{~m}}-\epsilon_{1, \mathrm{~m} \mp 1 / 2}^{+}$and $\Delta_{|2, \mathrm{~m}\rangle \rightarrow|1, \mathrm{~m} \mp 1 / 2\rangle^{-}}=\epsilon_{2, \mathrm{~m}}-\epsilon_{1, \mathrm{~m} \mp 1 / 2}^{-}$.
In order to calculate the averages in equation (11) and (12) we introduce three new Green functions which are given by

$$
\begin{aligned}
& \left\langle\left\langle\mathrm{X}^{0 \bar{\sigma}} \mathrm{Y}^{\mathrm{m}, \mathrm{m} \pm 1} \mid \mathrm{c}_{\sigma}^{+}\right\rangle\right\rangle^{\mathrm{r}}=\frac{\alpha_{\mathrm{m} \pm 1 / 2}^{\mp}\left\langle\mathrm{X}^{\sigma \bar{\sigma}} \mathrm{Y}^{\mathrm{m}, \mathrm{m} \pm 1}\right\rangle-\beta_{\mathrm{m} \pm 1 / 2}\left(\mathrm{P}_{0 \mathrm{~m}}+\mathrm{P}_{\sigma \mathrm{m}}\right)}{\left.\omega-\Delta_{\mid 1, \mathrm{~m} \pm 1 / 2}\right\rangle^{+} \rightarrow|0, \mathrm{~m}\rangle^{-\Sigma_{0}}} \\
& +\frac{\alpha_{\mathrm{m} \pm 1 / 2}^{ \pm}\left\langle\mathrm{X}^{\sigma \bar{\sigma}_{\mathrm{Y}} \mathrm{m}, \mathrm{m} \pm 1}\right\rangle+\beta_{\mathrm{m} \pm 1 / 2}\left(\mathrm{P}_{0 \mathrm{~m}}+\mathrm{P}_{\sigma \mathrm{m}}\right)}{\omega-\Delta_{|1, \mathrm{~m} \pm 1 / 2\rangle^{-} \rightarrow|0, \mathrm{~m}\rangle^{-\Sigma_{0}}}}
\end{aligned}
$$

$\left\langle\left\langle X^{0 \sigma} Y^{m m} \mid X^{\bar{\sigma} 0} Y^{m \pm 1, m}\right\rangle\right\rangle^{r}$

$$
\begin{aligned}
& =\frac{\alpha_{\mathrm{m} \pm 1 / 2}^{ \pm}\left\langle\mathrm{X}^{\bar{\sigma} \sigma} \mathrm{Y}^{\mathrm{m} \pm 1, \mathrm{~m}}\right\rangle-\beta_{\mathrm{m} \pm 1 / 2}\left(\mathrm{P}_{0 \mathrm{~m}}+\mathrm{P}_{\bar{\sigma} \mathrm{m} \pm 1}\right)}{\omega-\Delta_{|1, \mathrm{~m} \pm 1 / 2\rangle^{+} \rightarrow|0, \mathrm{~m}\rangle}-\Sigma_{0}}
\end{aligned}
$$

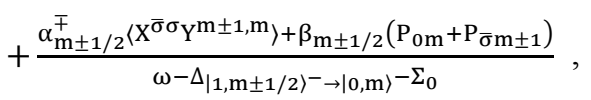

$\left\langle\left\langle X^{0 \bar{\sigma}} Y^{\mathrm{m}, \mathrm{m} \pm 1} \mid \mathrm{X}^{\bar{\sigma} 0} \mathrm{Y}^{\mathrm{m} \pm 1, \mathrm{~m}}\right\rangle\right\rangle^{\mathrm{r}}$

$$
\begin{gathered}
=\frac{\alpha_{\mathrm{m} \pm 1 / 2}^{\mp}\left(\mathrm{P}_{0 \mathrm{~m}}+\mathrm{P}_{\bar{\sigma} \mathrm{m} \pm 1}\right)-\beta_{\mathrm{m} \pm 1 / 2}\left\langle\mathrm{X}^{\bar{\sigma} \sigma} \mathrm{Y}^{\mathrm{m} \pm 1, \mathrm{~m}}\right\rangle}{\omega-\Delta_{|1, \mathrm{~m} \pm 1 / 2\rangle^{+} \rightarrow|0, \mathrm{~m}\rangle}-\Sigma_{0}} \\
+\frac{\alpha_{\mathrm{m} \pm 1 / 2}^{ \pm}\left(\mathrm{P}_{0 \mathrm{~m}}+\mathrm{P}_{\bar{\sigma} \mathrm{m} \pm 1}\right)+\beta_{\mathrm{m} \pm 1 / 2}\left\langle\mathrm{X}^{\bar{\sigma} \sigma} \mathrm{Y}^{\mathrm{m} \pm 1, \mathrm{~m}}\right\rangle}{\omega-\Delta_{|1, \mathrm{~m} \pm 1 / 2\rangle^{-} \rightarrow|0, \mathrm{~m}\rangle^{-\Sigma_{0}}}} .
\end{gathered}
$$

Thus the averages required to calculate the Green functions can be expressed as

$$
\begin{gathered}
P_{\sigma \mathrm{m}}=-\pi^{-1} \int \mathrm{d} \omega \overline{\mathrm{f}}(\omega) \operatorname{Im}\left\langle\left\langle\mathrm{X}^{0 \sigma} \mathrm{Y}^{\mathrm{mm}} \mid \mathrm{c}_{\sigma}^{+}\right\rangle\right\rangle^{\mathrm{r}} \\
\left\langle\mathrm{X}^{\sigma \bar{\sigma}} \mathrm{Y}^{\mathrm{m}, \mathrm{m} \pm 1}\right\rangle=-\pi^{-1} \int \mathrm{d} \omega \overline{\mathrm{f}}(\omega) \operatorname{Im}\left\langle\left\langle\mathrm{X}^{0 \bar{\sigma}} \mathrm{Y}^{\mathrm{m}, \mathrm{m} \pm 1} \mid \mathrm{c}_{\sigma}^{+}\right\rangle\right\rangle^{\mathrm{r}} \\
\left\langle\mathrm{X}^{\bar{\sigma} \sigma} \mathrm{Y}^{\mathrm{m} \pm 1, \mathrm{~m}}\right\rangle \\
=-\pi^{-1} \int \mathrm{d} \omega \overline{\mathrm{f}}(\omega) \operatorname{Im}\left\langle\left\langle\mathrm{X}^{0 \sigma} \mathrm{Y}^{\mathrm{mm}} \mid \mathrm{X}^{\bar{\sigma} 0} \mathrm{Y}^{\mathrm{m} \pm 1, \mathrm{~m}}\right\rangle\right\rangle^{\mathrm{r}} \\
\mathrm{P}_{\bar{\sigma} \mathrm{m} \pm 1} \\
=-\pi^{-1} \int \mathrm{d} \omega \overline{\mathrm{f}}(\omega) \operatorname{Im}\left\langle\left\langle\mathrm{X}^{0 \bar{\sigma}} \mathrm{Y}^{\mathrm{m}, \mathrm{m} \pm 1} \mid \mathrm{X}^{\bar{\sigma} 0} \mathrm{Y}^{\mathrm{m} \pm 1, \mathrm{~m}}\right\rangle\right\rangle^{\mathrm{r}},( \\
\delta_{\sigma} \mathrm{P}_{2 \mathrm{~m}}=-\pi^{-1} \int \mathrm{d} \omega \overline{\mathrm{f}}(\omega) \operatorname{Im}\left\langle\left\langle\mathrm{X}^{\bar{\sigma} 2} \mathrm{Y}^{\mathrm{mm}} \mid \mathrm{c}_{\sigma}^{+}\right\rangle\right\rangle^{\mathrm{r}}
\end{gathered}
$$

with $\bar{f}(\omega)=\left(f_{L}(\omega) \Gamma_{L}+f_{R}(\omega) \Gamma_{R}\right) /\left(\Gamma_{L}+\Gamma_{R}\right)$. To date, the Green functions (11)-(15) and the related averages (16)-(20) have formed a set of close equations, which can be used to calculate the charge and heat currents. When small bias voltage $\Delta \mathrm{V}$ and temperature gradient $\Delta \mathrm{T}$ are applied to the system, in the linear response regime, the charge current I and heat current $\mathrm{I}_{\mathrm{Q}}$ can be expressed as

$$
\begin{gathered}
\mathrm{I}=\mathrm{e}^{2} \mathrm{~L}_{0} \Delta \mathrm{V}+\frac{\mathrm{e} \mathrm{L}_{1}}{\mathrm{~T}} \Delta \mathrm{T}, \\
\mathrm{I}_{\mathrm{Q}}=-\mathrm{eL}_{1} \Delta \mathrm{V}-\frac{\mathrm{L}_{2}}{\mathrm{~T}} \Delta \mathrm{T},
\end{gathered}
$$

where $\mathrm{L}_{\mathrm{n}}=-\frac{1}{\mathrm{~h}} \sum_{\sigma} \int(\omega-\mu)^{\mathrm{n}} \frac{\partial \mathrm{f}}{\partial \omega} \Gamma \operatorname{ImG}_{\sigma}^{\mathrm{r}}(\omega) \quad(\mathrm{n}=0,1,2)$.

In the following calculation, the temperature and chemical potential satisfy $\mathrm{T}_{\mathrm{L}}=\mathrm{T}_{\mathrm{R}}=\mathrm{T}, \mu_{\mathrm{L}}=\mu_{\mathrm{R}}=\mu$ in two metallic electrodes. The linear electrical conductance can be calculated by the formula $G=\mathrm{e}^{2} \mathrm{~L}_{0}$ and the thermal conductance is written as $\kappa=\mathrm{T}^{-1}\left[\mathrm{~L}_{2}-\mathrm{L}_{1}^{2} \mathrm{~L}_{0}^{-1}\right]$. The ther- 
mopower $\mathrm{S}_{\mathrm{e}}$ is defined as the voltage drop $\Delta \mathrm{V}$ generated by the temperature gradient $\Delta \mathrm{T}$, i.e., $\mathrm{S}_{\mathrm{e}}=\Delta \mathrm{V} / \Delta \mathrm{T}=$ $-\mathrm{L}_{1}\left(\mathrm{eTL}_{0}\right)^{-1}$. The energy-conversion efficiency of the system is measured by the dimensionless figure of merit $\mathrm{ZT}=\mathrm{GS}_{\mathrm{e}}^{2} \mathrm{~T} /\left(\kappa_{\mathrm{ph}}+\kappa\right)$, where $\kappa_{\mathrm{ph}}$ represents the phonon thermal conductance. In the following calculation, we ignore the influence of $\kappa_{\mathrm{ph}}$ because $\kappa_{\mathrm{ph}}$ is suppressed in our structure.

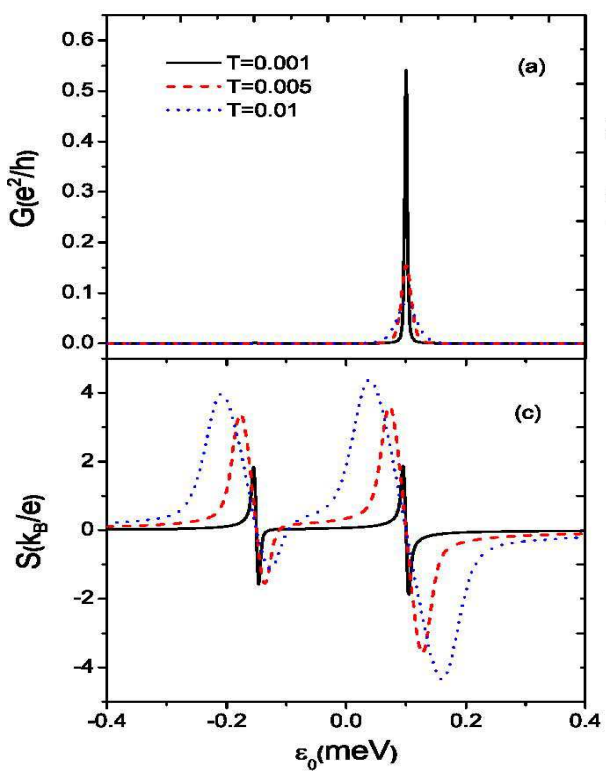

\section{Numerical Results}

Now we investigate numerically the thermoelectric properties of the system. Consider the symmetric contact between the single molecular magnet and the electrodes, i.e., $\Gamma_{\mathrm{L}}=\Gamma_{\mathrm{R}}=\Gamma$. In the linear regime, the relevant parameters are chosen as: the local spin $S=2, \mu_{\mathrm{L}}=\mu_{\mathrm{R}}=\mu=$ $0, \mathrm{~T}_{\mathrm{L}}=\mathrm{T}_{\mathrm{R}}=\mathrm{T}$.

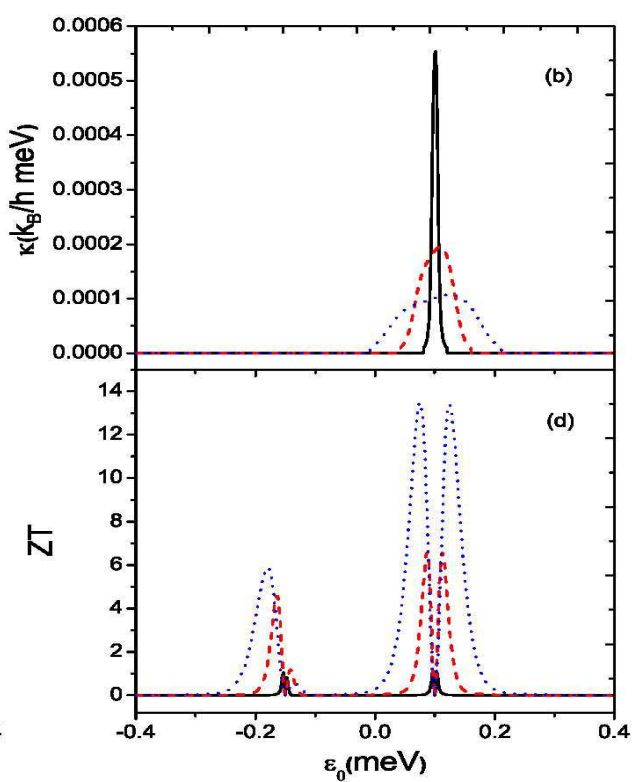

Figure 1. (Color online) (a) Electrical conductance, (b) thermal conductance, (c) thermopower, and (d) figure of merit as functions of the level position for the different temperature. The parameters are chosen as $U=0, J=0.1 \mathrm{meV}$ and $\Gamma=0.001 \mathrm{meV}$.
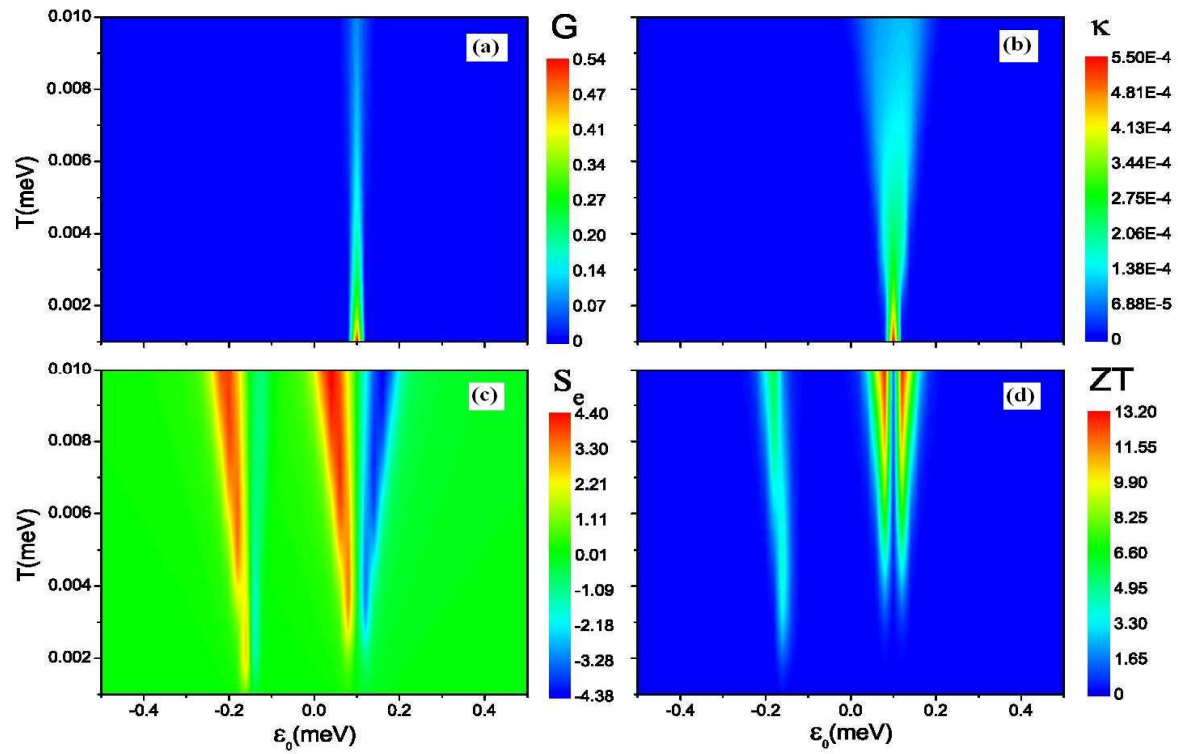

Figure 2. (Color online) Temperature and level position dependencies of the thermoelectric quantities: (a) electrical conductance, (b) thermal conductance, (c) thermopower, and (d) the figure of merit. The other parameters are the same as in Figure 1.

For the case that Coulomb interaction energy is infinite $(\mathrm{U} \rightarrow \infty)$, the doubly-occupied case is forbidden and there are only three energy levels of the molecular states due to the degeneracy, which form two effective transport channels:

$$
\begin{aligned}
& \mathrm{E}_{1}=\epsilon_{1, \mathrm{~m}}^{+}-\epsilon_{0, \mathrm{~m}}=\epsilon_{0}+\frac{\mathrm{J}}{2}(\mathrm{~S}+1), \\
& \mathrm{E}_{2}=\epsilon_{1, \mathrm{~m}}^{-}-\epsilon_{0, \mathrm{~m}}=\epsilon_{0}-\frac{\mathrm{J}}{2} \mathrm{~S} .
\end{aligned}
$$


The two channels lie on the bonding and anti-bonding combinations of $|\downarrow\rangle \otimes|\mathrm{m}+1 / 2\rangle$ and $|\uparrow\rangle \otimes|\mathrm{m}-1 / 2\rangle$. Figure 1 shows the electric conductance $G$, the thermal conductance $\kappa$, the thermopower $S_{e}$ and the figure of merit ZT as functions of the level position $\epsilon_{0}$ for the different temperature, in which $\epsilon_{\mathbf{0}}$ can be changed by tuning the gat voltage. Since the electrons entering the molecule favor forming the boding state and the value of $\Gamma$ is very small, as a result, as shown in figure 1(a), at the low temperature the electric conductance $G$ exhibits one evident peak only near bonding state level $\left(\epsilon_{0}=0.1 \mathrm{meV}\right)$ while near anti-bonding state level $\left(\epsilon_{0}=-0.15 \mathrm{meV}\right)$ one almost can not see the peak in the conductance. With the temperature increasing the peak gets sharper. The thermal conductance $\kappa$ has a similar behavior to the electric conductance, which is shown in figure 1(b). The property of thermopower $S_{e}$ is shown in figure 1(c); one can obviously see that oscillation and sign reversal in the thermopower spectrum. The sign of the thermopower is determined by the main carriers in transport. If the main carriers are electrons (holes), the thermopower is negative (positive). Although the electric conductance peak is not evident near anti-bonding state level, the evident asymmetry peak of thermopower can be observed near anti-bonding state. The thermoelectric efficiency of the system, described with the dimensionless figure of merit $\mathrm{ZT}$, is determined by the electrical conductance $\mathrm{G}$, thermal conductance $\kappa$ and thermopower $S_{\mathrm{e}}$. Figure 1(d) shows the figure of merit $\mathrm{ZT}$ as a function of the level position $\epsilon_{\mathbf{0}}$. Besides the typical double-peak structure the value of ZT near bonding state level can be more larger than the value of the conventional bulk materials. For example, at $\mathrm{T}=0.01 \mathrm{meV}$ (corresponding temperature is $0.1 \mathrm{~K}$ ), the value of $\mathrm{ZT}$ can reach 13. To show these properties more explicitly, we present the temperature and level position dependencies of the thermoelectric quantities in figure

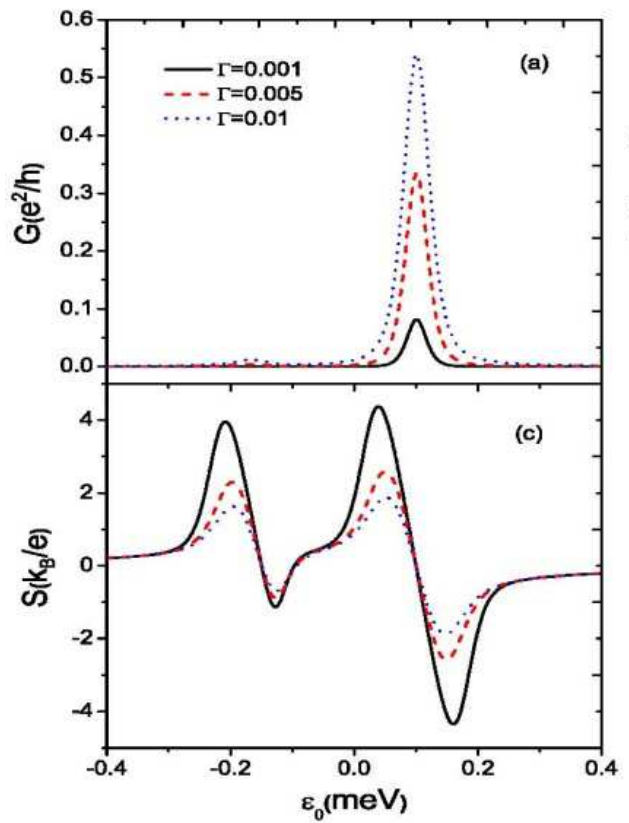

2. The eximious thermoelectric efficiency results from the electron resonance tunneling through the molecule state level.

In the regime of the sequential tunneling the coupling intensity between the electrodes and the molecule state level $\Gamma$ plays key role in determing thermoelectric properties. The influence of coupling intensity $\Gamma$ on the thermoelectric efficient is shown in figure 3 . At the low temperature, with $\Gamma$ increasing, the electric and thermal conductance peaks can also be observed near anti-bonding state level. Moreover, the electric and thermal conductance peaks near bonding state level get not only high, but also wider due to the broadening of molecule state level induced by the coupling between the electrodes and molecule state level (see figure 2(a) and figure 2(b)). The thermopower and the figure of merit are shown in figure $2(\mathrm{c})$ and $2(\mathrm{~d})$. For the large $\Gamma$ $(\sim 0.01 \mathrm{meV})$, the thermal conductance is large while the value of the thermopower is relatively small, which leads to small value of figure of merit near bonding state level. With $\Gamma$ decreasing, the thermal conductance get small while the value of the thermopower is relatively large, as a result, the figure of merit presents a very large value near bonding state level. A more general coupling intensity and level position dependencies of the thermoelectric quantities are shown in figure 4 . It can be clearly seen from figure 4 that $\mathrm{S}_{\mathrm{e}}$ and $\mathrm{ZT}$ are significantly enhanced for small coupling intensity $(\Gamma \ll T)$. It is obvious that in order to obtain large $\mathrm{ZT}$, besides requiring the device to work near bonding state level the match between the temperature and the coupling intensity is also important. In figure 5 we show the temperature and coupling intensity dependencies of figure of merit at $\epsilon_{0}=0.075 \mathrm{meV}$ (corresponding to the level position of a peak in ZT spectrum), which may be used to optimize the parameter region of $\mathrm{ZT}$.

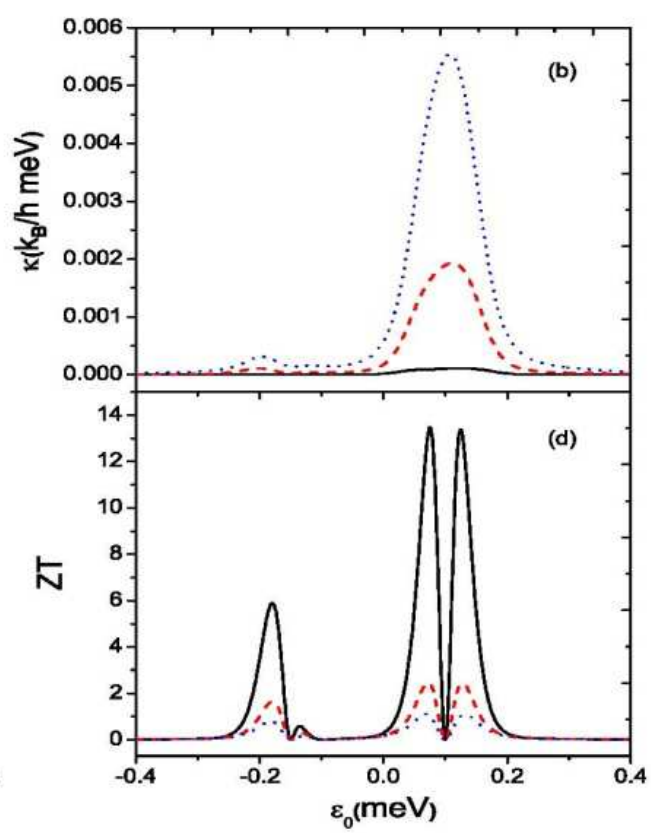

Figure 3. (Color online) (a) Electrical conductance, (b) thermal conductance, (c) thermopower, and (d) the figure of merit versus the level position for the different coupling intensity $\Gamma$ with $T=0.01 \mathrm{meV}$. The other parameters are the same as in Figure 1. 


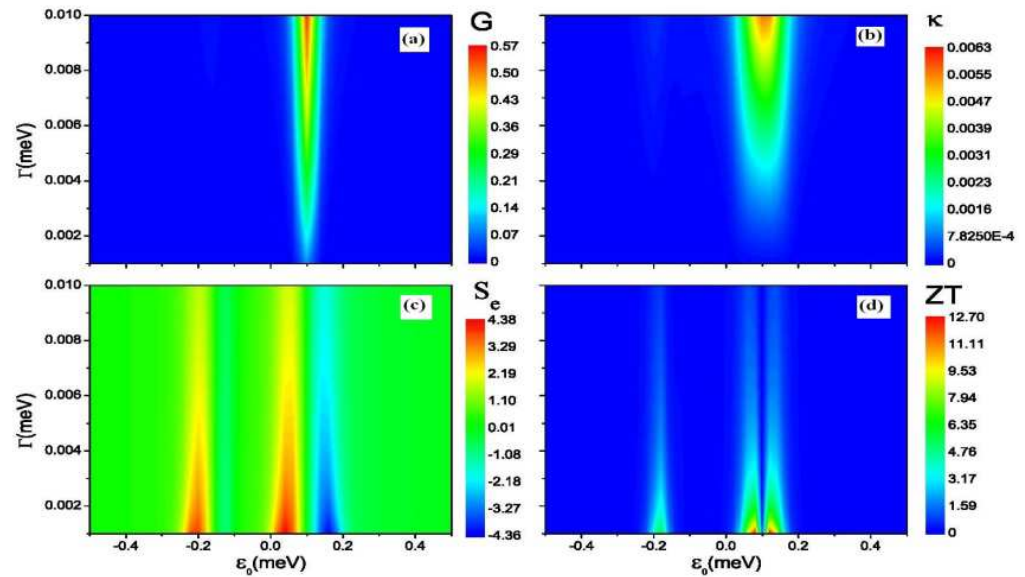

Figure 4. (Color online) (a) Electrical conductance, (b) thermal conductance, (c) thermopower, and (d) figure of merit as functions of the level position and coupling intensity for $T=0.01 \mathrm{meV}$. The other parameters are the same as in Figure 1.

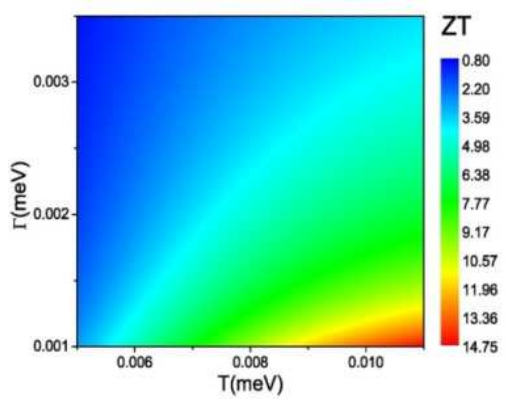

Figure 5. (Color online) Temperature and coupling intensity dependencies of figure of merit at $\epsilon_{0}=0.075 \mathrm{meV}$ (which corresponds to the level position of a peak in ZT spectrum). The other parameters are the same as in Figure 1.

Now we consider the impact of the finite Coulomb interaction on the thermoelectric properties in the regime of the sequential tunneling. For the finite Coulomb interaction, besides two effective transport channels(equation (23) and (24)) contributed by singly-occupied state the two new effective transport channels appear due to the presence of the doubly-occupied case, i.e.,

$$
\begin{gathered}
E_{3}=\epsilon_{2, m}-\epsilon_{1, m}^{+}=\epsilon_{0}+U-\frac{J}{2}(S+1), \\
E_{4}=\epsilon_{2, m}-\epsilon_{1, m}^{-}=\epsilon_{0}+U+\frac{J}{2} S .
\end{gathered}
$$

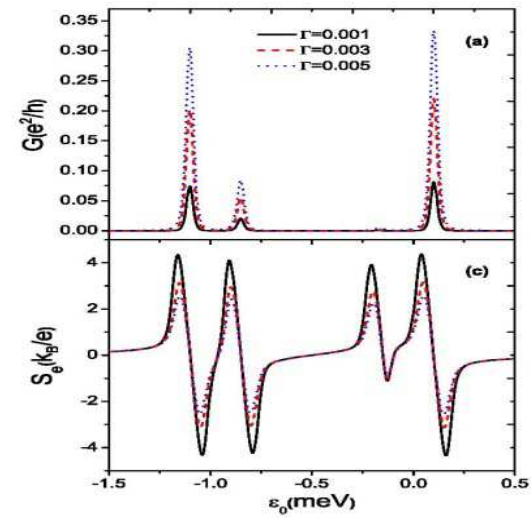

Figure 6. (Color online) (a) Electrical conductance, (b) thermal conductance, (c) thermopower, and (d) figure of merit as functions of the level position for $U=1 \mathrm{meV}$ and $T=0.01 \mathrm{meV}$. The other parameters are the same as in Figure 1.
At low temperature the Coulomb interaction splits the electric and thermal conductance spectra into two sets, which leads to the richer structure of the thermopower and the figure of merit. In figure 6 we show the relevant thermoelectric quantities as functions of the level position $\epsilon_{0}$ for $U=1 \mathrm{meV}$. It can be found from figure $6(\mathrm{a})$ and $6(\mathrm{~b})$ that four resonance peaks of $G$ and $\kappa$ correspond to four effective transport channels. The properties of the electric and thermal conductances corresponding to two sinly-occupied transport channels $\left(E_{1}, E_{2}\right)$ have been discussed above. For two peaks corresponding to doubly-occupied transport channels $\left(E_{3}, E_{4}\right)$, it is obvious that the peak near $E_{3}$ is lower than that near $\mathrm{E}_{4}$. This is because the contribution of the doubly-occupied transport channel on the transport depends on the transition probability of the molecule state from doubly-occupied state $|2, \mathrm{~m}\rangle$ to singly-occupied state $|1, \mathrm{~m}\rangle^{ \pm}$. The transition probability is proportionate to the empty probability of the state $|1, \mathrm{~m}\rangle^{ \pm}$. Since the electrons entering the molecule favor forming the bonding state, as a result the unoccupied probability of the state $|1, \mathrm{~m}\rangle^{+}$is less than that of the state $|1, \mathrm{~m}\rangle^{-}$, which results in the low conductance peak near $E_{3}$. However, the difference does not be found in $\mathrm{S}_{\mathrm{e}}$ and ZT. From figure 6(c) one can see that near $E_{3}$ and $E_{4}$ not only the thermopower is almost uniform, but also the figure of merit is almost uniform and its value can be much larger than unity.

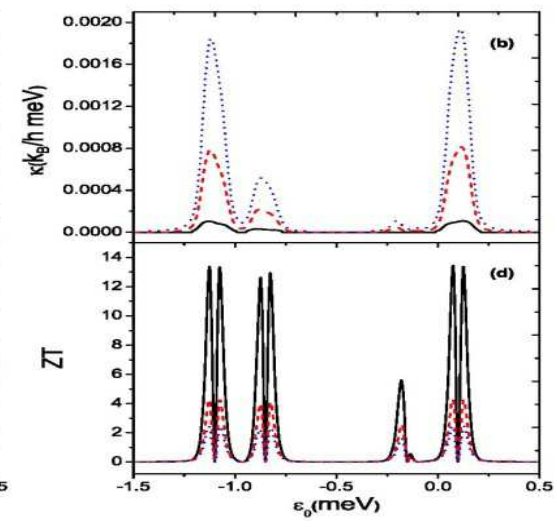




\section{Conclusions}

In summary, we study thermoelectric properties of an isotropic SMM coupled to two metal electrodes in the linear response regime. For the infinite Coulomb interaction the singly-occupied states determine the effective transport channels. At the low temperature the electric and thermal conductance exhibits one evident peak only near bonding state level for the small coupling intensity. Accordingly, the thermopower and the figure of merit present the large values near bonding state level. For the given temperature the thermopower and the figure of merit are significantly enhanced with coupling intensity decreasing $(\Gamma \ll T)$. When the Coulomb interaction is finite, the spectra of thermoelectric quantities split into two sets. The peaks of the thermopower and figure of merit corresponding to doubly-occupied transport channels are much larger than unity for the small coupling intensity. Moreover an optimized parameter region of $\mathrm{ZT}$ is given.

\section{Acknowledgements}

This work was supported by the National Nature Science Foundation of China (Grants No. 11274208).

\section{References}

[1] G. Jeffrey Snyder and E. S. Toberer, "Complex thermoelectric materials" Nature Mater. , Vol.7, pp.105, 2008.

[2] M.Cutler and N.F.Mott, "Observation of Anderson Localization in an Electron Gas", Phys. Rev. Vol. 181, pp.1336, 1969.

[3] B. Kubala, J. Konig, and J. Pekola, "Violation of the Wiedemann-Franz Law in a Single-Electron Transistor ", Phys. Rev. Lett., Vol. 100, pp. 066801, 2008.

[4] J. Liu, Q. F Sun, and X. C. Xie, "Enhancement of the thermoelectric figure of merit in a quantum dot due to the Coulomb blockade effect ",Phys. Rev. B., Vol. 81, pp. 245323, 2010 .

[5] Y. Dubi and M. D.Ventra, "Colloquium: Heat flow and thermoelectricity in atomic and molecular junctions", Phys. Mod. Rev., Vol.83, pp.131, 2011.

[6] M. Turek and K. A. Matveev, "Cotunneling thermopower of single electron transistors", Phys. Rev. B., Vol. 65, pp. 115332,2002 .

[7] J. Koch, F. von Oppen, Y. Oreg, and E. Sela, "Thermopower of single-molecule devices", Phys. Rev. B., Vol. 70, pp. 19510, 2004

[8] B. Kubala and J. Konig, "Quantum-fluctuation effects on the thermopower of a single-electron transistor",Phys. Rev. B., Vol. 73, pp.195316,2006.

[9] X. Zianni, "Coulomb oscillations in the electron thermal conductance of a dot in the linear regime",Phys. Rev. B ., Vol. 75, pp.045344, 2007.
[10] D. Boese and R. Fazio, "Thermoelectric effects in Kondo -correlated quantum dots”,Europhys. Lett. , Vol. 56, pp. 576, 2001 .

[11] B. Dong and X. L. Lei, "Effect of the Kondo correlation on the thermopower in a quantum dot",J. Phys.: Condens. Matter ., Vol. 14, pp. 11747, 2002.

[12] M. Krawiec and K. I. Wysokinski, "Thermoelectric effects in strongly interacting quantum dot coupled to ferromagnetic leads”,Phys. Rev. B .,Vol. 73, pp.075307, 2006.

[13] R. Sakano, T. Kita, and N. Kawakami, "Thermopower of Multiorbital Kondo Effect via Single Quantum Dot System at Finite Temperatures",J. Phys. Soc. Jpn. 76, pp. 074709, 2007.

[14] R. Scheibner, H. Buchmann, D. Reuter, M. N. Kiselev, and L. W. Molenkamp, "Thermopower of a Kondo Spin- Correlated Quantum Dot”,Phys. Rev. Lett.,Vol. 95, pp. 176602, 2005.

[15] M. Yoshida and L. N. Oliveira, "Thermoelectric effects in quantum dots ", Physica B., Vol. 404, pp.3312, 2009.

[16] P.Trocha and J.Barnas, "Large enhancement of thermoelectric effects in a double quantum dot system due to interference and Coulomb correlation phenomena", Phys. Rev. B., Vol.85, pp. 085408, 2012.

[17] C. M. Finch, V. M. Garcia-Suarez, and C. J. Lambert, "Giant thermopower and figure of merit in single-molecule devices",Phys. Rev. B ., Vol.79, pp.033405, 2009.

[18] Y. S. Liu and X. F. Yang, "Enhancement of thermoelectric efficiency in a double-quantum-dot molecular junction", J. Appl. Phys., Vol.108, pp. 023710, 2010.

[19] O. Karlstrom, H. Linke, G. Karlstrom, and A. Wacker,"Increasing thermoelectric performance using coherent transport”, Phys. Rev. B., Vol.84, pp.113415, 2011.

[20] D. Segal,"Thermoelectric effect in molecular junctions: A tool for revealing transport mechanisms", Phy. Rev. B., Vol.72, pp. 165426, 2005.

[21] B. Ludoph and J. M. van Ruitenbeek, "Thermopower of atomic-size metallic contacts", Phys. Rev. B., Vol. 59 , pp.12290, 1999.

[22] P. Reddy, S. Y. Jang, R. A. Segalman, and A. Majumdar, "Thermoelectricity in Molecular Junctions" , Science Vol.315, pp.1568, 2007,

[23] S. K. Yee, J. A. Malen, A. Majumdar, and R. A. Segalman, "Thermoelectricity in Fullerene-Metal Heterojunctions", Nano Lett., Vol.11, pp.4089, 2011.

[24] J. P. Bergfield and C.A. Stafford,"Thermoelectric Signatures of Coherent Transport in Single-Molecule Heterojunctions", Nano Lett., Vol. 9, pp.3072, 2009.

[25] R. Q. Wang, L. Sheng, R. Shen, B. Wang, and D. Y. Xing, "Thermoelectric Effect in Single-Molecule-Magnet Junctions", Phy. Rev. Lett ., Vol.105, pp.057202, 2010.

[26] Z. Zhang, L. Jiang, R. Wang, B. Wang, and D. Y. Xing, "Thermoelectric-induced spin currents in single-molecule magnet tunnel junctions", Appl. Phy. Lett., Vol. 97, pp. 242101,2010 . 
[27] P. S. Cornaglia, G. Usaj, and C. A. Balseiro, "Tunable charge and spin Seebeck effects in magnetic molecular junctions", Phy. Rev. B., Vol. 86, pp.041107(R), 2012.

[28] C. Timm, F. Elste, "Spin amplification, reading, and writing in transport through anisotropic magnetic molecules", Phys. Rev. B., Vol. 73, pp.235304, 2006.

[29] Y. Meir, N.S. Wingreen, "Landauer formula for the current through an interacting electron region", Phys. Rev. Lett., Vol. 68 , pp.2512, 1992.

[30] H. Haug, A. Jauho, Quantum Kinetics in Transport and Optics of Semiconductors, Springer-Verlag, Berlin, 1996.
[31] Peng-Bin Niu, Yu-Ying Zhang, Qiang Wang, Yi-Hang Nie, "Quantum transport through anisotropic molecular magnets: Hubbard Green function approach", Phys. Lett. A ., Vol.376, pp.1481, 2012.

[32] Qing-feng Sun, Hong Guo, "Double quantum dots: Kondo resonance induced by an interdot interaction", Phys. Rev. B., Vol. 66, pp.155308, 2002 .

[33] M. Tolea, B.R. Bułka, "Theoretical study of electronic transport through a small quantum dot with a magnetic impurity", Phys. Rev. B., Vol. 75, pp.125301, 2007. 